\title{
Temporal Stability of a Transparent Mode Group Diversity Multiplexing Link
}

\author{
C. P. Tsekrekos, Student Member, IEEE, M. de Boer, A. Martinez, Member, IEEE, F. M. J. Willems, Fellow, IEEE, \\ and A. M. J. Koonen, Senior Member, IEEE
}

\begin{abstract}
The temporal behavior of a mode group diversity multiplexing (MGDM) link is examined. MGDM has been proposed as a way of creating parallel, independent communication channels over multimode fiber (MMF) links using different groups of modes. When the effect of dispersion can be neglected, the output electrical signals of an MGDM link are related to the input ones via a real-valued transmission matrix. The transmission matrix is vulnerable to changes in the modal spectrum of the propagating mode groups as well as to the coupling of light at the input and output of the MMF. Measurements of the transmission matrix of a two-input two-output system over 12.7 h show that an MGDM link can be very stable.
\end{abstract}

Index Terms-Graded-index multimode fiber (GI-MMF), mode group diversity multiplexing (MGDM), optical multiple-input multiple-output (MIMO), transparent optical link.

\section{INTRODUCTION}

M ULTIMODE fiber (MMF) is extensively used in in-building and campus networks. Its large core diameter makes it much easier to splice MMF than single-mode fiber. In the classical intensity-modulation direct-detection (IM-DD) single-input single-output approach, the multitude of propagating modes in the MMF is considered a restricting factor. In particular, it limits the bandwidth of such MMF links due to the modal dispersion caused by the differential propagation delay of the modes. However, the modal fields being orthogonal, the propagating modes are spatial degrees of freedom that could be exploited in an MMF link.

Mode group diversity multiplexing (MGDM) is an IM-DD multiple-input multiple-output (MIMO) technique that creates independent, transparent-regarding the transmission format-communication channels over MMF, using different subsets of propagating modes [1]. MGDM shares similarities with MIMO techniques used in wireless communications. At the transmitting side, $N$ sources are used to launch $N$ different groups of modes. At the output of the MMF, each of $M(M \geq N)$ photodetectors responds to a different combination of the optical power carried by the $N$ mode groups. The electrical signals after the photodetectors are demultiplexed

Manuscript received July 7, 2006; revised September 1, 2006. This work was supported by the Freeband Impulse Programme of the Ministry of Economic Affairs of The Netherlands.

The authors are with COBRA Research Institute, Eindhoven University of Technology, $5600 \mathrm{MB}$ Eindhoven, The Netherlands (e-mail: c.tsekrekos@tue.nl.)

Color versions of Figs. 1, 3, and 4 are available at http://ieeexplore.ieee.org.

Digital Object Identifier 10.1109/LPT.2006.886978 with electrical signal processing. Therefore, no signal orthogonality is required at the optical intensity domain.

Factors such as temperature changes, wavelength drifting, or mechanical vibrations may change the modal spectrum of the propagating mode groups, the launch conditions at the MMF input, and the coupling of the optical power to the $M$ photodetectors, therefore affecting the performance of the link. This letter addresses the issue of temporal stability of an MGDM link, showing that such a link can be very stable.

\section{TRANSMISSION MATRIX}

Propagation in the MMF introduces dispersion, both within and among the excited mode groups, as well as mode mixing and attenuation. In short-reach optical networks, the effect of dispersion can be neglected when the requirements on bandwidth are not very high. For fibers shorter than $100 \mathrm{~m}$, the bandwidth of the transmitted signals can still be in the range of a few gigahertz [2], [3]. In addition, mode mixing is very limited in silica MMFs [4]. Vector $\mathbf{s}_{R}(t)$ of the received electrical signals can be related to vector $\mathbf{s}_{T}(t)$ of the transmitted electrical signals via a real-valued $M \times N$ transmission matrix $\mathbf{H}(t)=\left\{h_{i, j}(t)\right\}$, i.e., $\mathbf{s}_{R}(t)=\mathbf{H}(t) \mathbf{s}_{T}(t)+\mathbf{x}(t)$, where $\mathbf{x}(t)$ is a noise term. The matrix elements express the signal mixing. A similar approach has been used in recent work on optical MIMO, namely dispersive multiplexing [5] and coherent optical MIMO [6], where a matrix is also used to relate the output signals to the input ones. However, these techniques use binary phase-shift keying signal format and complex-valued matrices.

When matrix $\mathbf{H}(t)$ is known, the input signals can be recovered by straightforward matrix inversion, a method consistent with the requirement of transparency [7]. The estimated transmitted signals are then $\hat{\mathbf{s}}_{T}(t)=\hat{\mathbf{H}}^{\dagger}(t) \mathbf{s}_{R}(t)+\mathbf{y}(t) \Rightarrow$

$$
\hat{\mathbf{s}}_{T}(t)=\hat{\mathbf{H}}^{\dagger}(t) \mathbf{H}(t) \mathbf{s}_{T}(t)+\hat{\mathbf{H}}^{\dagger}(t) \mathbf{x}(t)+\mathbf{y}(t)
$$

where $\hat{\mathbf{H}}^{\dagger}(t)$ is an estimate of a pseudoinverse of $\mathbf{H}(t)$ (or the inverse of $\mathbf{H}(t)$ in the case of an $N \times N$ system) and $\mathbf{y}(t)$ the noise from the demultiplexing circuit.

From (1), it is evident that any temporal variations of $\mathbf{H}(t)$, and consequently $\hat{\mathbf{H}}^{\dagger}(t)$, will change the noise level through the factor $\hat{\mathbf{H}}^{\dagger}(t) \mathbf{x}(t)$ and thus the power budget of the link. Furthermore, if the channel estimation is not ideal, i.e., $\hat{\mathbf{H}}^{\dagger}(t) \neq \mathbf{H}^{\dagger}(t)$, some crosstalk will remain among the channels, since the inner product $\hat{\mathbf{H}}^{\dagger}(t) \mathbf{H}(t)$ will not result in the $N \times N$ identity matrix.

\section{EXPERIMENTAL RESULTS}

Fig. 1 shows the experimental setup used to investigate the temporal behavior of a $2 \times 2 \mathrm{MGDM}$ link. A fiber concentrator 


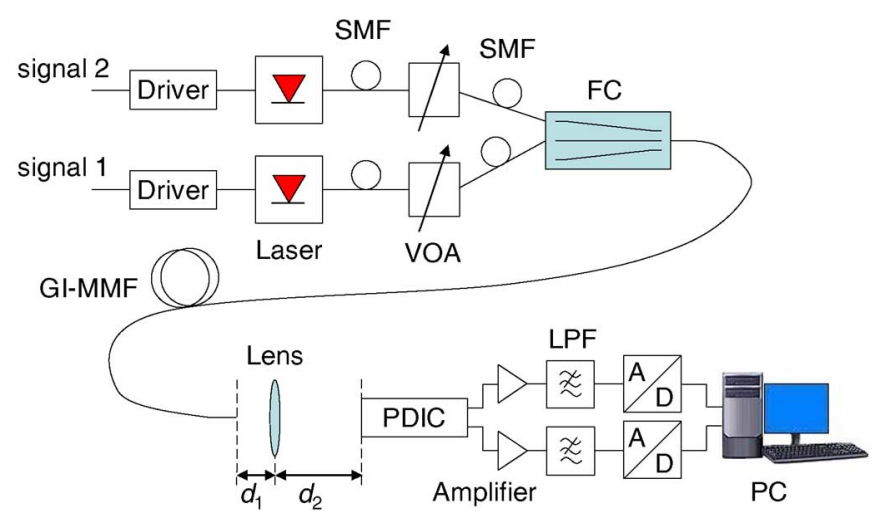

Fig. 1. Experimental setup for the investigation of a $2 \times 2$ MGDM link.

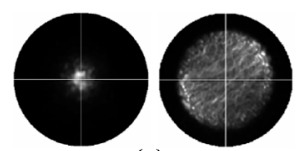

(a)

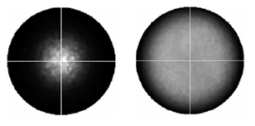

(b)

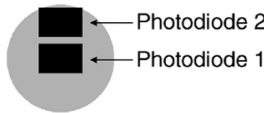

(c)
Fig. 2. NFP of the lower (left) and higher (right) order modes channel at the output of the (a) 10-m and (b) 2-km-long GI-MMF. (c) Receiving side of the setup when a lens is used to magnify the NFP by a factor of two; gray color indicates the area of the magnified NFP of the higher order modes channel and black the PDIC photodiodes.

(FC) was used to simultaneously excite two groups of modes. The FC is a planar waveguide that transforms a linear array of waveguides with a single-mode cutoff around $1200 \mathrm{~nm}$ from the standard pitch of $125 \mu \mathrm{m}$ into $30 \mu \mathrm{m}$. Two uncooled directly modulated 635-nm Fabry-Pérot multiquantum-well laser diodes were connected to two adjacent ports of the FC. In this way, light could be launched into thick core graded-index (GI) MMFs with $0-\mu \mathrm{m}$ (Transmitter 1) and 30- $\mu \mathrm{m}$ (Transmitter 2) radial offset from the fiber axis. The level of the optical power was controlled by variable optical attenuators. At the receiving side of the link, a lens projected the near-field pattern (NFP) at the GI-MMF output onto an integrated linear array of photodiodes followed by preamplifiers (PDIC) [8]. The PDIC has 11 photodiodes of rectangular shape of $80 \times 120 \mu \mathrm{m}$ and highest responsivity at visible light. The distances of the lens from the end facet of the fiber $\left(d_{1}\right)$ and the PDIC $\left(d_{2}\right)$ were such as to magnify the NFP by a proper factor $(m)$, in order to allow two out of the 11 PDIC photodiodes to be employed, as illustrated in Fig. 2(c). The two electrical outputs of the PDIC were followed by a transimpedance amplifier and a low-pass filter and then connected to a personal computer through an analog-to-digital converter for further electrical processing.

A 10-m-long silica GI-MMF with core-cladding diameter $185 / 250 \mu \mathrm{m}$, and a 2-km-long 148/200 $\mu \mathrm{m}$ one were tested. The link was then studied under two extreme conditions. In the 10-m fiber, limited mode mixing takes place and the NFP at the fiber output shows strong speckle contrast. In the case of the 2-km fiber, there is mode mixing and the intensity distribution of the NFP is smoother [9]. Fig. 2(a) and (b) shows the NFP at the output facet of the two GI-MMFs as observed with a charged-coupled device camera through a microscope $(20 \times$, 0.40 numerical aperture). Each launched mode group yields a distinguishable NFP for both GI-MMFs. The size of the NFP imposed that $m \approx 2$.

One way to determine the real-valued $h_{i, j}(t)$ is to assign a sinusoidal pilot tone at each MGDM transmitter and measure the level (voltage) of these pilot tones at the output of each photodiode. Following this method, we used 7- and 8-MHz sinusoids to directly modulate the laser of Transmitter 1 and 2 , respectively. For example, the level of the 7-MHz tone at the electrical output of the photodiode of Receiver 2 gives the value of $h_{2,1}(t)$. The level of the pilot tones at the receiving side of the link was measured by performing a fast Fourier transform of the received electrical signals. In particular, approximately 2.5 measurements per second were taken, with an integration time of $82 \mu$ s for each one.

Fig. 3 shows the measured $h_{i, j}(t)$ during a period of $12.7 \mathrm{~h}$ for the two GI-MMFs, together with temperature measurements in the same period. $h_{i, j}(t)$ is normalized to the average $\left\langle h_{1, j}(t)+\right.$ $\left.h_{2, j}(t)\right\rangle$ in the 12.7 -h period, which is proportional to the average total received power emitted by the $j$ th laser. In this way, the value of $h_{i, j}(t)$ expresses the spatial diversity irrespective of the average received power. It is evident that $h_{i, j}(t)$ varies moderately over time. Long-term fluctuations of the $h_{i, j}(t)$ coefficients can be associated with the corresponding temperature changes. This is very clear in the case of the 2-km-long GI-MMF. Apart from the mode distribution and the coupling at the input and output of the GI-MMF, temperature variations affect the optical power emitted by the lasers, since no power stabilization was used. The effect of the temperature can be reduced by using connectorized components at the input and output of the GI-MMF as well as an electrical feedback loop to control the optical power emitted by the lasers.

In general, it is difficult to explain thoroughly the variations in the diagrams of Fig. 3, due to the multitude of factors involved. The significance of the results lies in the observed stability of the link. Taking into account that our link does not use MGDM-specific components, only better performance should be expected in an optimally designed link.

\section{Electrical Crosstalk After DemultipleXing}

The $h_{i, j}(t)$ coefficients can be used to estimate the electrical crosstalk between the two MGDM channels, assuming matrix inversion as the demultiplexing algorithm. If $\mathbf{G}(t)=\hat{\mathbf{H}}^{\dagger}(t) \mathbf{H}(t)=\left\{g_{i, j}(t)\right\}$, the electrical crosstalk $C_{1}(t)$ and $C_{2}(t)$ at Channels 1 and 2, respectively, is defined in decibels as

$$
\begin{aligned}
& C_{1}(t)=20 \log _{10}\left|\frac{g_{1,2}(t)}{g_{1,1}(t)}\right| \\
& C_{2}(t)=20 \log _{10}\left|\frac{g_{2,1}(t)}{g_{2,2}(t)}\right| .
\end{aligned}
$$

When both the channel estimation and the matrix inversion are ideal, crosstalk is equal to $-\infty$.

To give a figure of merit of how fast the channel matrix changes, we can calculate the electrical crosstalk for different periods of the channel estimation $(\Delta t)$. Specifically, we assume that the measured normalized $\mathbf{H}(t)$ is ideal and we calculate $\hat{\mathbf{H}}^{\dagger}(t)$ only once every $\Delta t$. Crosstalk is then estimated by (2). Fig. 4 shows the average and maximum calculated crosstalk 

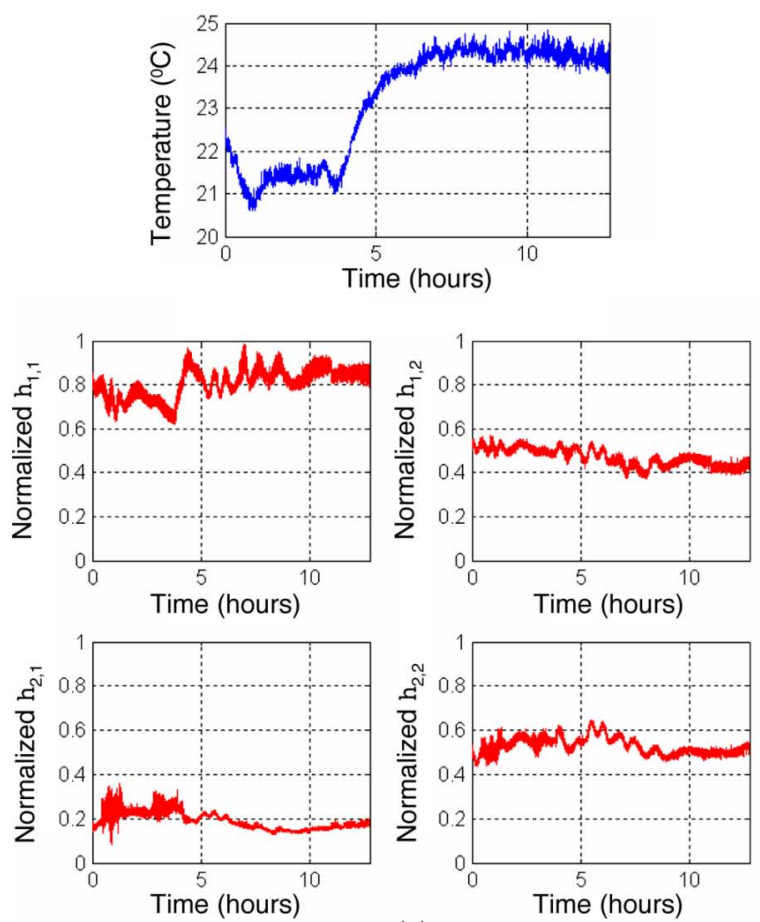

(a)
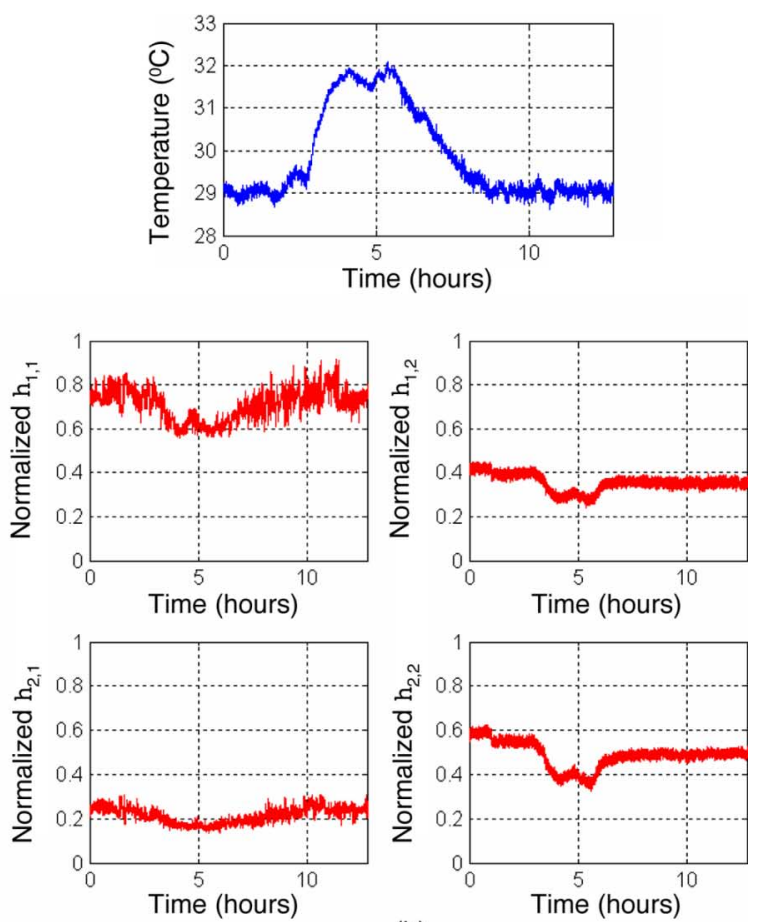

(b)

Fig. 3. Temporal variations of the transmission matrix elements $h_{i, j}$ when (a) the 10-m and (b) the 2-km GI-MMF is used, together with temperature measurements during the same period. The value of $h_{i, j}(t)$ is normalized to the average $\left\langle h_{1, j}(t)+h_{2, j}(t)\right\rangle$.

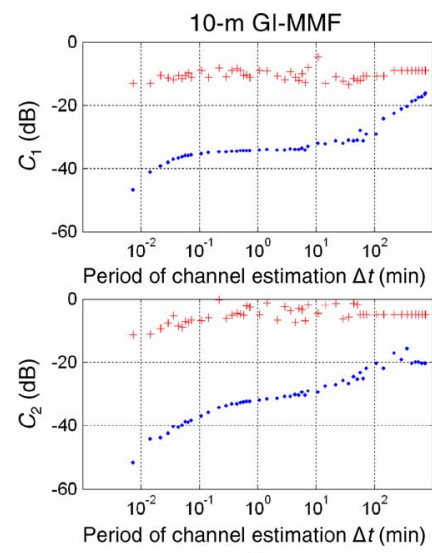

(a)
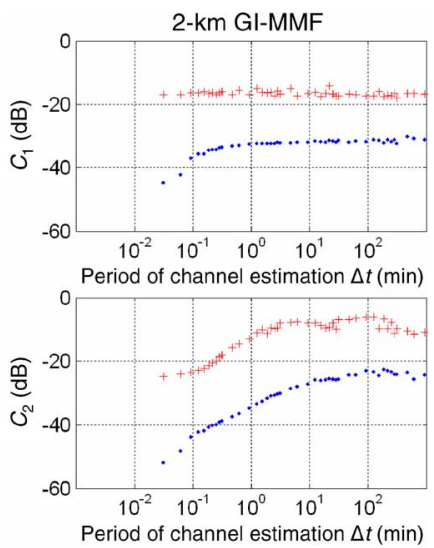

(b)
Period of channel estimation $\Delta t(\mathrm{~min})$

Fig. 4. Calculated average $(\cdot)$ and maximum $(+)$ electrical crosstalk versus the time period $\Delta t$ of the channel estimation during the $12.7 \mathrm{~h}$ where $h_{i, j}(t)$ were measured for (a) the 10-m and (b) the 2-km GI-MMF. Matrix inversion was assumed to demultiplex the received electrical signals.

at the two MGDM channels for different $\Delta t$ during the 12.7-h measurement of the $h_{i, j}(t)$ coefficients. Crosstalk clearly depends on the channel estimation period. In a complete MGDM link that includes both the optical and electrical units, $\Delta t$ as well as the the power level of the pilot tones will be determined by experimentation taking into account the application with the highest requirements on signal-to-noise ratio.

\section{CONCLUSION}

In this letter, we have presented measurements of the realvalued transmission matrix of a two-input two-output MGDM link. A $2 \times 2$ experimental setup has been realized and the link has been shown to be very stable both when a short $(10 \mathrm{~m})$ and a long $(2 \mathrm{~km})$ silica GI-MMFs were used.

\section{ACKNOWLEDGMENT}

The authors would like to thank Dr. A. M. van der Lee (Philips Research Laboratories) for providing the PDIC.

\section{REFERENCES}

[1] T. Koonen, H. P. A. van den Boom, I. T. Monroy, and G. D. Khoe, "High capacity multi-service in-house networks using mode group diversity multiplexing," in Proc. OFC, Los Angeles, CA, Feb. 22-27, 2004, Paper FG4.

[2] C. Carlsson, A. Larsson, and A. Alping, "RF transmission over multimode fibers using VCSELs-comparing standard and high-bandwidth multimode fibers," J. Lightw. Technol., vol. 22, no. 7, pp. 1694-1700, Jul. 2004.

[3] L. Raddatz, I. H. White, D. G. Cunningham, and M. C. Nowell, "An experimental and theoretical study of the offset launch technique for the enhancement of the bandwidth of multimode fiber links," J. Lightw. Technol., vol. 16, no. 3, pp. 324-331, Mar. 1998.

[4] S. Kawakami and H. Tanji, "Evolution of power distribution in gradedindex fibres," Electron. Lett., vol. 19, no. 3, pp. 100-102, Feb. 3, 1983.

[5] H. R. Stuart, "Dispersive multiplexing in multimode optical fiber," Science, vol. 289, pp. 281-283, Jul. 14, 2000.

[6] A. R. Shah, R. C. J. Hsu, A. Tarighat, A. H. Sayed, and B. Jalali, "Coherent optical MIMO (COMIMO)," J. Lightw. Technol., vol. 23, no. 8, pp. 2410-2419, Aug. 2005.

[7] J. R. Barry, E. A. Lee, and D. G. Messerschmitt, Digital Communications. Norwell, MA: Kluwer, 2004.

[8] G. W. de Jong, J. R. M. Bergervoet, J. H. A. Brekelmans, and J. F. P. van Mil, "A DC-to-250 MHz current pre-amplifier with integrated photo-diodes in standards CBiMOS, for Optical-Storage Systems," in Proc. ISSCC, San Francisco, CA, Feb. 4-6, 2002, Paper 21.8.

[9] A. M. J. Koonen, "Bit-error-rate degradation in a multimode fiber optic transmission link due to modal noise," IEEE J. Sel. Areas Commun., vol. SAC-4, no. 9, pp. 1515-1522, Dec. 1986. 